\title{
Modeling and Simulation of Intelligent Vision Based Pattern-Recognition of Colour-Iris
}

\author{
Pongthep Kulchatchai ${ }^{\text {a,b }}$ and Amir Anvar ${ }^{\text {a }}$ \\ ${ }^{a}$ School of Mechanical Engineering, The University of Adelaide, South Australia 5005 \\ Emails: pongthep.kulchatchai@student.adelaide.edu.au,amir.anvar@adelaide.edu.au \\ ${ }^{b}$ School of Industrial Technology, Uttaradit Rajabhat University, Thailand \\ Email: pongthep.k@uru.ac.th
}

\begin{abstract}
Among the several algorithms that exist for Iris pattern recognition, Daugman's innovation is one of the most implemented approaches. His algorithm is based on Near-InfraRed (NIR) images which have been proven as an effective technique particularly when used in controlled scenarios [1]. The trend of using gray NIR-base images then dominates the process of Iris recognition and influences the majority of eyes' images databases. The aim of this paper is to create a model that achieves a more effective Iris-recognition system (i.e. Iris encoding and recognition) by considering the individualities of both the pattern-structure and Colour of the Iris-pattern. The conducted simulation shows positive results. Via colour analysis, the model can efficiently and accurately divide all suspect samples into a small group. The recognition system then searches and compares within a smaller quantity of suspected samples. This case has shown that the model can increase the recognition speed up to $70 \%$ [6]. In the proposed circumstances, this model also supports the accuracy of Iris recognition.
\end{abstract}

Keywords: Pattern Recognition, Local Analysis, Global Analysis, Colour Iris. 


\section{Background}

Iris recognition is one method of biometric authentication that can recognise a person's identity. The effectiveness of Iris recognition has been extensively verified [1-5 and 7-9]. The shape of Iris' structures was the key to identifying the pattern of the Iris necessary to perform recognition (Figure 1). Numerically and graphically, these inimitable structures are interpreted as having varied intensities and magnitudes of digital data. Therefore, several techniques have been developed to analyse the pattern data. For instance, Daugman [1] applied the principle of Fourier transform to execute 2-D Gabor filter for creating the individual frequency and orientation of Iris' data. Wildes [2] used the Laplacian Pyramid method to decompose the size of the original data and in turn applied normalized correlation to combine and create distinctive data. These two methods investigate and analyse the data of NearInfraRed NIR images. The main reason for using NIR for iris recognition is because NIR wavelengths will not be absorbed by the melanin pigment in either light or dark Irises and thus, will transfer most information of the Iris' structures back to camera [3]. It can therefore be inferred from the previous research that NIR influences the majority of Iris recognition but neglects the colour features.

Visible light can be also used effectively to capture Iris patterns by fusing data in Red-Green-Blue (RGB) colour system channels. This fusion uses the

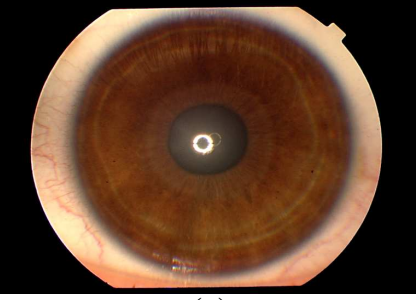

(a)

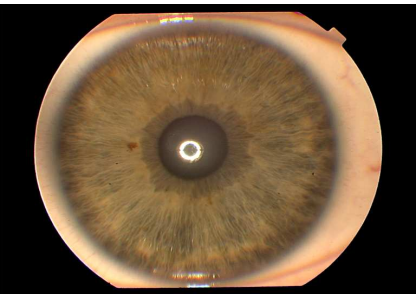

(b)

Figure 1. Two samples of different eyes colour, Iris patterns [6]. leverages from the broad spectrum of visible light. This is due to the spectrum highlighting and the different information via eye-colour [5]. Experiments and studies [4,5 and 8] on the multichannel data show that, the fusion of multichannel data can increase the effectiveness of recognition up to the same level of that by NIR wavelength. The recognition mentioned in this paragraph investigates the pattern of Iris' structures. Alternatives to using NIR include the utilization of visible light and fusion techniques.

The colour of an Iris pattern has its discriminative potential. Two eyes with significant difference in colour of Iris patterns can be differentiated easily without consideration of their Iris pattern structures. However, identifying an eye in various samples by colour alone is not recommended as an effective method. This paper investigates a method of using Iris pattern colour to increase the effectiveness and accuracy of recognition based-on comparison of coloured Iris' structures sample(s) against of reference sample.

\section{Overview}

Generally, the Iris recognition process is made up of four steps: Iris image Acquisitions, Iris image Preprocessing, Iris Encoding and Recognition. In this paper, several methods are used to present the results. In addition, the Novelty of using Iris pattern with its natural colour is achieved with Iris-encoding and pattern-recognition steps. The objective of the recognition model is to combine the individualities of both structure and colour of Iris. In this research, a version of an Iris recognition model is developed and successfully simulated using MATLAB workspace.

\section{Methodology}

\subsection{Image Acquisition}

A typical method of capturing the Iris colour images is complex [4 and 5] and different from using NIR [1 and 2] technique. As part of the investigation reported here, to prove the validity of adopted techniques, an available online database of eye pattern images was used [6]. The database includes 128 different Iris samples from 64 peoples (left and right eye of each person counts as two different eye samples: because each Iris pattern is different). Each three images corresponding to the left and three images corresponding to the right eye were taken from each individual. All the images were saved in 24-bit RGB, PNG format with resolution 576X768 pixels. The images exclude most of the sclera area for experimental purposes (see Figure 1). In this experiment, there were 3 Iris images in each sample extracted from a large database. Two out of 3 images from each Iris were selected to create two sampling sections. The first section was used as a reference data. The second section was used as the test data. 


\subsection{Image Preprocessing}

The purpose of image preprocessing is to exclude noise (e.g., pupil, sclera, eyelid, eyelash and other external noises such as light reflection) from the testing subject to ensure that only Iris patterns remain. Initially, the model uses the Hough transform technique on edge images [2] in order to locate the inner and outer boundary of the Iris (Figure 2a). It was found that the accuracy and speed of the localization method could be improved considerably by estimating the parameters of the pupil [7] in the red channel. Then, the parameters are used to find the outer boundary in blue channel [8]. In relation to the other noise, the testing images were not corrupted by eyelid or eyelash and had no significant light reflection. Therefore, this particular topic of noise can be discarded from the simulation. Finally, by using the rubber sheet model [1], the Iris pattern after the localization was remapped from the original circular arrangement to a rectangular arrangement (Figure $2 b)$.

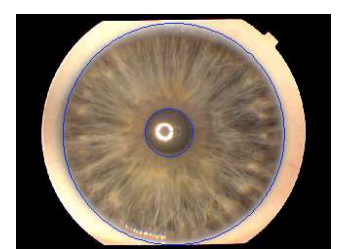

(a)

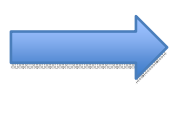

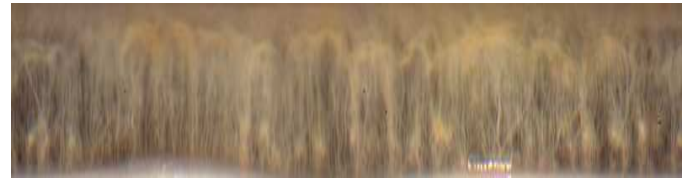

(b)

Figure 2. Iris Image Processing (a) Iris localization image and (b) Rectangular arrangement of the pattern.

\subsection{Iris Encoding}

Generally, the purpose of Iris encoding is to extract the magnitude and intensity of information from Iris patterns. An effective method requires the ability to accurately differentiate the magnitude values and their intensities between different locations. The encoded data is then stored in an efficient way to support the speed and data capacity. In order to facilitate the local analysis, a method developed by Daugman was tested and used. Statistically, there is always a possibility of the same patterns in different Iris. However, the local analysis with a sufficient size of data may decrease the probability of the agreement within the comparison of eye samples and thus, will increase its recognition accuracy. For example, the Daugman technique encodes Iris information into 2048 bits phase; which approximately equals a probability of " 249 tosses of a fair coin" [1]. Consequently, the local recognition method is an established technique and has a number of advantages [1, 4-5and 8].

The Global analysis also has some advantages including its capability to reduce the noises' prominence by using a data combination technique. A good example of a global algorithm comes from the Wildes method that decomposes Iris images into 64 sizes of distinctive value [2]. During the experiment, the test [9] showed that the accuracy of the global method is lower than the local method. Therefore, to maximize the effectiveness of Iris recognition, local analysis is essential.

Although global analysis alone is not always attractive, an innovative model in this paper analyzes the difference of Iris colour using global information. The model is then able to efficiently and accurately exclude a small group of suspicious samples from all the samples before executing local analysis.

\subsubsection{Local Analysis on the Structure of Iris Pattern}

To clarify local analysis utilizing Daugman's algorithm, information from the red channel was used. The experiment [4] showed that recognition in the red channel could achieve higher accuracy than using the blue or green channels. As presented in Figure 3, after the image is processed via 2-D Gabor filter, a bit-templatepattern appears with the signs of Negative $=0$ and Positive $=1$.

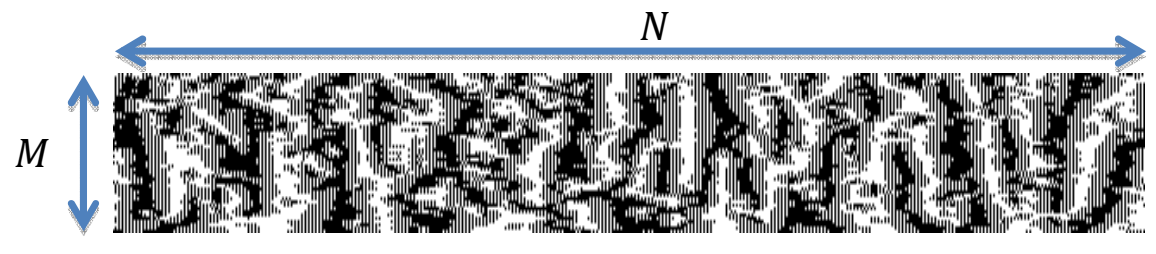

Figure 3. A sample of an Iris template. 


\subsubsection{Global Analysis on Colour of Iris}

To conduct a global analysis, a preprocessed image was compressed using the Gaussian Pyramid technique that was a built in MATLAB function. This technique was also used in WILDES [2]. In order to decompose the image, the information was averaged corresponding to the lower level of the Pyramid and thus, significant information was preserved. The simulation for the model presented in this paper used the Gaussian process aiming to reduce the target size to 1 pixel for each RGB colour channel which is represented on the top section of the Figure 4.

The next step of this process is to find the relationship between three-fixed values - the magnitude of the colour value laid from $0-1$, corresponding to the values in the range $0-255$. The second-order polynomial was used to find three coefficients between these values. From the experiment, when comparing the two different sample' sessions signifying that the same eyes were captured in different times, the agreement of the coefficient in the first and second order is consistent but fluctuated in the zero order (Figure 5). The correlation coefficient of the comparison between the two sessions of 128 different Iris eye samples equal to 0.9982 , 0.9979 and 0.9302 for the second, first and zero order respectively. In conclusion, the relation of the threecolour pixels is significantly correlated and consistent from eye to eye.

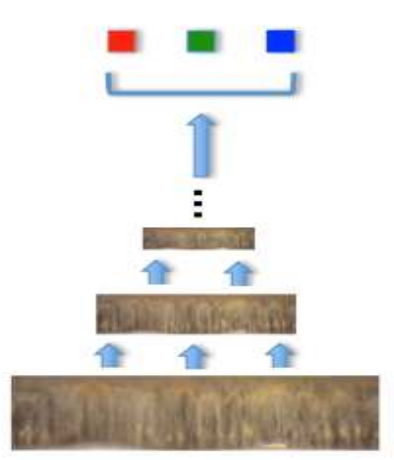

Figure 4. Image size reduction by factor of 2 using Gaussian Pyramid Method.

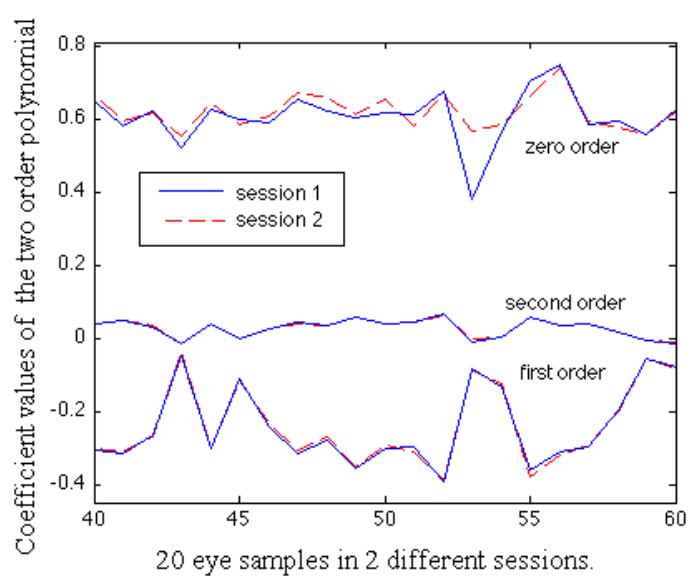

Figure 5. The agreement of each coefficient between two sample sessions.

\subsection{Recognition}

The aim of the recognition technique used in this investigation is to match and measure the similarity between two encoding data. Generally, test data will be checked with all the stored data to find the highest evaluated agreement or until the agreement reaches an expected value. In the model, before executing local analysis, the relation between the eye colours was used (from 3.3.2) to reduce a number of checking pairs. This allowed the simulation of the Iris recognition to become quicker in detecting the identification.

\subsubsection{Local Analysis on Structure Pattern of Iris}

The Hamming Distance (HD) number was used to measure the similarity between the two Iris pattern templates [1]. The value of the HD is laid from 0 to 1 . Zero HD means the comparison of two samples template (from 3.3.1) which is in perfect agreement.

\subsubsection{Global Analysis on Colour of Iris}

A range limit $\mathrm{R}$ of the first and second order coefficients (from 3.3.2) was used to divide the suspected samples into a small category. As shown in the results presented in Figure 6, when the model executed the Iris recognition, the total comparison of the sample number 50 was reduced from 20 to 7 samples. The comparison occurred only in the range limit that was approximately within the vicinity of $0.3121<\mathrm{R}<-0.2773$ (see figure 6). The zero coefficient order was discarded due to its low correlation.

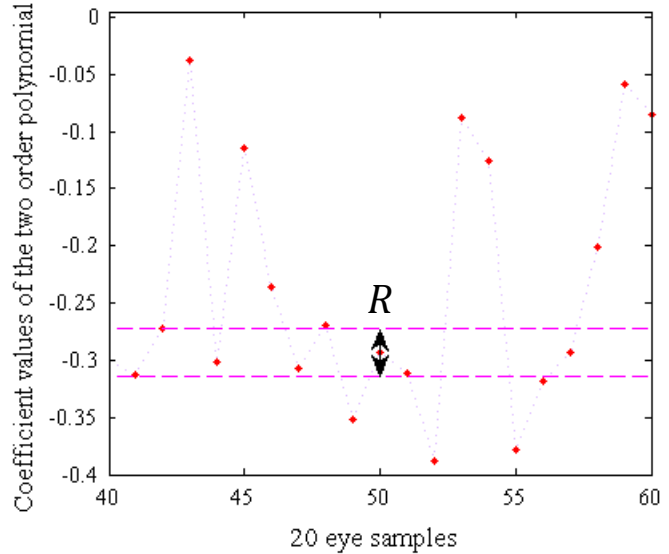

Figure 6. Diagram representing upper and lower limits for the first order coefficient of the $50^{\text {th }}$ sample 


\section{Model Simulation using Intelligent Method}

The simulation system procedure commenced with the data sampling and using Polynomial order 2 and 2-D Gabor filter to each sample. The outcomes of this segment were collected in a template. The system was then set the limit based on the results of Polynomial order 2. Furthermore, an automated comparison and decisionmaking process applied, using Fuzzy Logic technique. In addition of finding the lowest value of Hamming Distance to finalize the identification process. Figure 7 represents the steps of the Colour Iris pattern simulation model process.

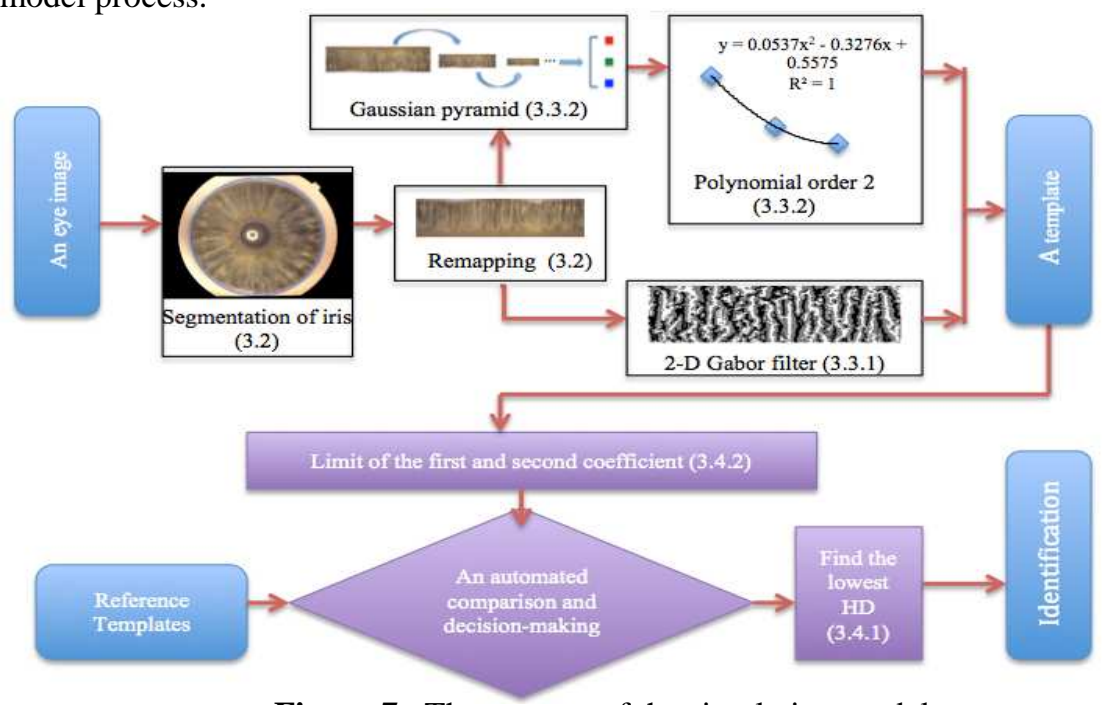

Figure 7. The process of the simulation model.

\section{Results and Discussion}

Table 1. The quantities of the checking pair within the Range limit R. (Without function of the global analysis, were counted 128 checking pairs per test)

\begin{tabular}{|c|c|c|c|c|c|c|c|c|c|}
\hline \multirow{3}{*}{$\begin{array}{c}\text { The index number } \\
\text { of the Input eye } \\
\text { sample }\end{array}$} & \multicolumn{9}{|c|}{ The number of the checking pair in the Range limit $\mathbf{R}$ with different safety factor $S *$} \\
\hline & \multicolumn{3}{|c|}{$\begin{array}{l}\text { Filter by the first coefficient } \\
\text { (B)*** }\end{array}$} & \multicolumn{3}{|c|}{$\begin{array}{l}\text { Filter by the second coefficient } \\
\text { (A)*** }\end{array}$} & \multicolumn{3}{|c|}{ Filter by both coefficient $* *$} \\
\hline & $\mathrm{S}=1$ & $\mathrm{~S}=1.5$ & $\mathrm{~S}=2$ & $\mathrm{~S}=1$ & $\mathrm{~S}=1.5$ & $\mathrm{~S}=2$ & $\mathrm{~S}=1$ & $\mathrm{~S}=1.5$ & $\mathrm{~S}=2$ \\
\hline 1 & 20 & 32 & 37 & 14 & 21 & 28 & 12 & 21 & 28 \\
\hline 38 & 26 & 33 & 44 & 38 & 45 & 46 & 23 & 33 & 43 \\
\hline 52 & 6 & 12 & 20 & 6 & 12 & 19 & 4 & 8 & 17 \\
\hline 104 & 32 & 38 & 43 & 28 & 36 & 40 & 26 & 36 & 40 \\
\hline $\begin{array}{l}\text { Average of } 128 \\
\text { samples }\end{array}$ & 22.63 & 31.41 & 38.9 & 23.88 & 33.11 & 40.15 & 17.68 & 27.4 & 35 \\
\hline
\end{tabular}

* $\mathrm{S}=1$ means $\mathrm{R}$ (form 3.4.2) is set equal to the highest possible difference used to filter the same Iris in two sessions (in this experiment, $\mathrm{R}=0.0084$ and 0.0348 for second and first coefficient respectively). Therefore, in the Table $1, \mathrm{~S}=1$ refers to the least range limit $\mathrm{R}$ that filter the sample without an error.

** The samples are within the range limit if both values of the first and the second coefficient fit within their $\mathrm{R}$ range limit.

$* * *$ For 256 data, (A): $\operatorname{mean}=0.0188, \max =0.064, \min =-0.0165$. (B): mean $=-0.2052, \max =-0.0214, \min =-0.3931$.

The model was designed to automaticaly compare and identify the source. The reference would have a deduction number of suspected samples ranging up to $86 \%$ (as presented in Table 1, the average number with $\mathrm{S}=1$ and decision adopted by both coefficient). The simulation in table 2 compared all the Iris templates of the first session with the second session to find the minimum HD number. In the small template size, the result from Table 2 shows that, the proposed model could reduce the number of unsuccessful recognitions. The proposed model can filter unwanted samples that have a possibility of imposture templates that share a similarity with the genuine Iris template. When the template size was increased, the error can be reduced to zero. However, during the identification process, the proposed model simulated faster than the normal method by $70 \%$. 
With consideration that light-intensity could affect the rate of success of this model, the experiment was conducted by capturing three individual eyes (Figure 8) with and without a flashlight. Six images were tested in the model. The results showed that the model can correctly categorize the sample when the safety factor $\mathrm{S}=2$ was applied. The category aided in identifying the correct person because the eye colour was notably different. The third person's eye was shown to be significantly dilated due to the use of the flash light, as shown in Figures 8(c) and 8(d). The dilatation reduced the accuracy of the local analysis considerably (HD = 0.423 , the identification is correct in this test but likely to be incorrect in future comparisons). In this case, the model can decrease the error of the Iris recognition when the comparison data-samples stored in the systems are increased.

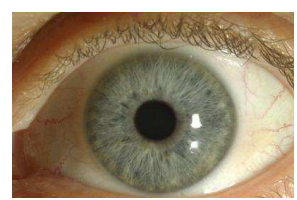

(a)

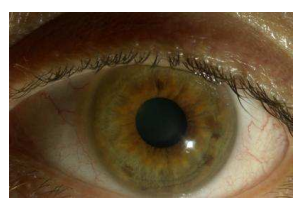

(b)

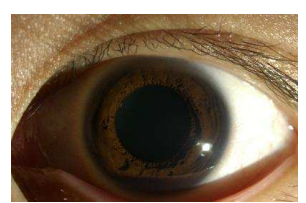

(c)

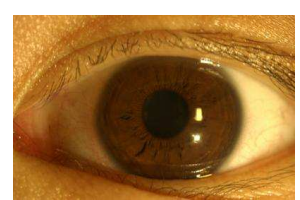

(d)

Figure 8. The samples of eyes images used to investigate the influence of light intensity on the model, (a), (b) and (c) are the left eye images of three persons that were captured with flashlight, (d) is an example of the same eye in (c) but were captured without flashlight.

\section{Conclusion}

In this paper, Iris recognition and its application using the local analysis of eye pattern structure and global analysis of eye colour was discussed. The local analysis employed the algorithm developed by Daugman [1]. Whereas, the global analysis uses the correlation between the three-fix values of Colour channels RGB to separate the eyes colour, Iris patterns into several categories. The result of simulation using Iris colour pattern recognition showed the reduction of processing time by $70 \%$. Additionally, the outcomes of the tests also performed that the error reduction in the small scale of the Iris template is positive.

\section{Acknowledgements}

The authors would like to thank Prof. Bob Casson, Mr. Alan Hoane and the staff at the Ophthalmology Clinic of the Royal Adelaide Hospital (RAH) for their support in providing the sampling equipment. The Authors would also like to acknowledge Mr. Amir Parsa Anvar for his editorial comments on this paper.

\section{References}

1. Daugman, J 2004, 'How Iris Recognition Works', IEEETrans.CSVT2004, vol.14, issue. 1, pp. 21-30.

2. WILDES, RP 1997, 'Iris recognition: an emerging biometric technology', IEEE, vol. 85, issue 9, pp. $1348-1363$.

3. Daugman, J 2006, 'Identifying Persons by Their Iris patterns', in FORENSIC HUMAN IDENTIFICATION: An introduction, eds T Thompson \& S Black, Boca Raton, pp. 271-285.

4. Vatsa, M, Singh, R, Ross, A, Noore, A 2010, 'Quality-Based Fusion for Multichannel Iris Recognition', 20th International Conference on Pattern Recognition, pp.1314-1317.

5. Boyce, C, Ross, A, Monaco, M, Hornak, L, Li, X 2006,"Multispectral Iris analysis: A preliminary study," in Proceedings of the 2006 Conference on Computer Vision and Pattern Recognition Workshop.

6. Michal Dobeš and Libor Machala, Iris Database, http://www.inf.upol.cz/iris/.

7. Feng, X, Fang, C, Ding, X, Wu, Y 2006, 'Iris Localization with Dual Coarse-to-fine Strategy', The 18th International Conference on Pattern Recognition (ICPR'06), Department of Electronic Engineering, Tsinghua University, Beijing 100084, P. R. China.

8. Sun, C, Melgani, F, Francesco, DN, Zhou, C, Zhang, L, Liu, X 2008, 'Incremental Learning Based Colour Iris Recognition', micai, 2008 Seventh Mexican International Conference on Artificial Intelligence, pp.319-324.

9. Ma, L, Tan, T, Wang, Y, Zhang, D 2004, 'Efficient Iris Recognition by Characterizing Key Local Variations' IEEE TRANSACTIONS ON IMAGE PROCESSING, vol. 13, no. 6.

10. Masek, L, Kovesi. P 2003, MATLAB Source Code for a Biometric Identification System Based on Iris Patterns. The School of Computer Science and Software Engineering, The University of Western Australia. 